\title{
Diffusion Kurtosis-Based Brain Image Segmentation for the Structural Remodeling of White Matter in Patients with Hypoxic-Ischemic Encephalopathy
}

\author{
Yongkun Kang $(\mathbb{D}$, Chuang Sun $(\mathbb{D}$, Chao Yang $(\mathbb{D}$, and Honghai Chen $(\mathbb{D})$ \\ Department of Radiology, The Second Affiliated Hospital of Dalian Medical University, Dalian, 116023, China \\ Correspondence should be addressed to Honghai Chen; 201812212502011@zcmu.edu.cn
}

Received 12 July 2021; Revised 4 August 2021; Accepted 11 August 2021; Published 31 August 2021

Academic Editor: Gustavo Ramirez

Copyright ( $\odot 2021$ Yongkun Kang et al. This is an open access article distributed under the Creative Commons Attribution License, which permits unrestricted use, distribution, and reproduction in any medium, provided the original work is properly cited.

\begin{abstract}
The aim was to explore the application value of brain image segmentation algorithm based on diffusion kurtosis imaging (DKI) in the structural remodeling of white matter (WM) in patients with hypoxic-ischemic encephalopathy (HIE). 120 patients with leukoencephalopathy and hypoxic-ischemic encephalopathy were selected as the research objects. Their heads were scanned by conventional magnetic resonance imaging (MRI) and DKI sequence. Besides, DKI based on the image segmentation algorithm was applied to process DKI images, DKE software was employed to obtain the values of fractional anisotropy (FA) and mean kurtosis (MK), and the differences in FA and MK were compared between acute and chronic phases. The results showed that the proposed algorithm could realize the best values of Jaccard similarity (JS) and Dice similarity coefficient (DSC) in WM, gray matter (GM), and cerebrospinal fluid (CSF), and the segmentation accuracy was better than other algorithms. FA values at the acute and chronic phases were compared in the area around lesions ( $0.421 \pm 0.065$ vs. $0.454 \pm 0.052)$, the posterior limb of internal capsule on the affected side $(0.498 \pm 0.027$ vs. $0.504 \pm 0.046)$, and the pedunculus cerebri on the affected side $(0.558 \pm 0.038$ vs. $0.568 \pm 0.042)$, and the differences were statistically substantial $(P<0.05)$. Moreover, MK values at the acute and chronic phases were also compared in the area around lesions (1.362 \pm 0.098 vs. $1.407 \pm 0.077)$, the centrum semiovale on the affected side $(1.305 \pm 0.102$ vs. $1.343 \pm 0.076)$, the posterior limb of internal capsule on the affected side $(1.338 \pm 0.543$ vs. $1.382 \pm 0.076)$, and the pedunculus cerebri on the affected side $(1.329 \pm 0.089$ vs. $1.398 \pm 0.099)$, showing a statistical meaning $(P<0.05)$. The results indicated that the changes of FA and MK were related to the structural remodeling of WM. The DKI image segmentation algorithm could be applied in the diagnosis of leukoencephalopathy in patients with hypoxic-ischemic encephalopathy, and DKI technology was of great significance for the research of structural remodeling of WM.
\end{abstract}

\section{Introduction}

HIE is the most important cause of morbidity and mortality in full-term babies $[1,2]$ and is a brain injury caused by hypoxia in the perinatal period. If the disease is relatively mild, timely detection and treatment can achieve a better prognosis. However, HIE can easily lead to neurodevelopmental delay, cerebral palsy, hearing and visual impairment, and epilepsy if it is relatively severe [3]. At present, there is a high incidence of HIE among newborn babies in China. HIE is a great threat to the lives of newborn babies, which is related to the overall national quality. It has become a hot topic of medical research at home and abroad. Studies have found that HIE may also cause leukoencephalopathy [4]. Leukoencephalopathy is a general term for various WM lesions, most of which are caused by hypoxia and ischemia [5]. Therefore, timely diagnosis and treatment is of great significance for the prevention and treatment of HIE and leukoencephalopathy.

Imaging technology can observe human brain structure and various lesions based on three-dimensional perspective, which has an important advantage in the research of brain reorganization and remodeling. Computed tomography, ultrasound, and conventional MRI can help diagnose brain injury but cannot quantify WM injury $[6,7]$. Diffusion tensor imaging (DTI) is a noninvasive examination method, which can effectively observe, track, and clearly display the nerve fiber tracts of WM [8]. What is 
more, FA is commonly applied in DTI to represent the tropism degree of axons, microtubules, and myelin. It is also adopted to evaluate the damage degree of WM fibers. However, DTI has some limitations, for example, its sensitivity is not high and cannot be applied to evaluate the cross fiber bundle. On the contrary, DKI is a further optimization of DTI [9]. DTI analyzes the difference of human tissue structure based on the Gaussian diffusion model, while DKI applies the non-Gaussian diffusion model to evaluate the morphological and pathological changes of human tissue more accurately. DKI can obtain parameters such as apparent diffusion coefficient (ADC), FA, MK, radial kurtosis (RK), and axial kurtosis (AK). MK is the mean of diffusion kurtosis in all gradient directions and is highly sensitive to the perception of nerve fiber lesions. However, there are few reports about DKI in leukoencephalopathy and HIE. Image segmentation is a key step in brain image diagnosis technology. Brain structure is usually composed of WM, GM, and CSF, and the main segmentation target is to accurately segment the above three. Common image segmentation algorithms include fuzzy C-means (FCM), self-organizing mapping (SOM), fuzzy local information c-means clustering (FLICM) algorithm, genetic algorithm (GA), and random forest $[10,11]$. Due to the complexity of medical images, a single algorithm is unable to complete accurate segmentation, and usually multiple algorithms are combined for segmentation. Therefore, the FLICM algorithm and random forest classifier were combined to propose the brain image segmentation algorithm, which was used for the evaluation of leukoencephalopathy in patients with HIE. The structural recombination of leukoencephalopathy was investigated by analyzing the differences of FA and MK.

\section{Materials and Methods}

2.1. Research Objects and Grouping. A total of 120 patients with leukoencephalopathy and HIE were selected as the research objects admitted to hospital from September 2019 to October 2020. All subjects met diagnostic criteria for HIE and suffered from leukoencephalopathy, and there was an exclusion of patients with traumatic brain injury, cardiopulmonary, liver, and kidney dysfunction, and congenital genetic disease. This experiment had been approved by the Ethics Committee of hospital, and all the patients included in the experiment had known and agreed.

\subsection{Magnetic Resonance Imaging Detection. A Siemens} Verio 3.0T superconducting magnetic resonance scanner was employed to examine the heads of all subjects, and a 12channel cranial coil was also applied. Each patient was in supine position with the head advanced.

After scanning, DTI and color direction-coded DTI images were generated automatically. DKE software was adopted to further process the obtained DKI images, so as to gain the value graphs of FA and MK. The same patient was scanned twice, and the images were registered. The area of glial hyperplasia, posterior limb of internal capsule, corpus callosum (including splenium, body part, and genu), corona radiata, pedunculus cerebri, and bilateral centrum semiovale around the lesion were selected as the regions of interest for the FA value graph of the first scan. Then, Image J software was applied to automatically generate the MK value graph of the first scan and the FA and $\mathrm{MK}$ value graphs of the second scan in the regions of interest. In addition, $\mathrm{FA}$ and $\mathrm{MK}$ were recorded at the different parts of WM for two scans.

\subsection{Brain Diffusion Kurtosis Imaging-Based Image Segmen-} tation Algorithm. The brain DKI-based image segmentation algorithm proposed in this study mainly included two steps, namely, segmentation of $\mathrm{H}$-shape region of CSF in DKI images and segmentation of WM and GM in DKI images.

First, brain DKI images were obtained from the database, and there was the skull separation pretreatment. Then, the Fourier descriptor in the H-shape region of CSF was calculated. It was assumed that DKI image size was $A \times B$, so the coordinate of center pixel was $(A / 2, B / 2)$. The rectangular size was $A / 3 \times B / 3$, and the Canny algorithm [12] was used for the image edge extraction in the rectangular area. The gray histogram is further calculated, and the initial threshold value is the gray data with the lowest frequency. The gray value $>$ pixel gray of initial threshold value was set as 0 , and gray value $<$ pixel gray of initial threshold value was set as 1 , so as to obtain the preliminary segmentation image. Then, the Fourier descriptor of the initial segmentation image was calculated. The Fourier descriptor of the initial segmentation image was compared with the Fourier descriptor of the $\mathrm{H}$-shape region. If the Euclidean distance between the two was $<0.02$, the segmentation image was of $\mathrm{H}$ shape; otherwise, the threshold value was adjusted until the Euclidean distance $<0.02$ was met.

The FLICM algorithm [13] was used for the initial classification labels to pixels in other regions, and six eigenvalues of pixel points were calculated as follows.

The pixel gray value $(I)$ was expressed in the following equation:

$$
I=f(i, j),
$$

where $i$ and $j$ referred to the vertical and horizontal coordinate of the pixel of any point in the image.

The neighborhood means $(\mu)$ could be calculated as follows:

$$
\mu=\frac{1}{k^{2}} \sum_{i=0}^{k-1} \sum_{j=0}^{k-1} f(i, j) .
$$

The gradient $(G)$ could be calculated as the following equation:

$$
G(i, j)=\sqrt{(f(i+1, j)-f(i, j))^{2}+(f(i, j+1)-f(i, j))^{2}} .
$$

The neighborhood standard deviation (Std) could be calculated as follows: 


$$
\operatorname{Std}=\sqrt{\frac{1}{k^{2}} \sum_{i=0}^{k-1} \sum_{j=0}^{k-1}(f(i, j)-\mu)^{2}} .
$$

The calculation of local entropy $(E)$ is as follows:

$$
E=-\sum_{i=0}^{L-1} p_{i} \log _{2} p_{i}
$$

The MODE value referred to the grayscale value with the current pixel as the center and the highest frequency pixel in the $k \times k$ neighborhood near the center.

In the above equations, $f(I, j)$ stood for the sum of the gray values of pixels in the $k \times k$ neighborhood near the central pixel point; $k$ expressed the odd number; $C_{i}$ represented the number of pixels with the same gray value as pixel $i$ in the $k \times k$ neighborhood; and $L$ stood for the total number of pixels.

Then, the initial classification label and the calculated eigenvalues were input into the random forest classifier for normalization processing:

$$
D=\frac{D}{D_{(\max )}},
$$

where $D$ and $D_{\max }$ stand for the input and maximum eigenvectors, respectively.

Half of the image pixel points were regarded as training samples, and the other half were considered as test samples, which were input into the random forest classifier for testing, respectively, so that the segmentation results were obtained.

JS and DSC were adopted to evaluate the performance of the image segmentation algorithm. DSC was applied to evaluate the accuracy of the algorithm and describe the image coincidence degree, and JS ranged from 0 to 1 . The higher the JS, the better the coincidence between the segmentation and standard images:

$$
\begin{gathered}
\mathrm{JS}=\frac{\left|M_{i} \cap N_{i}\right|}{\left|M_{i} \cup N_{i}\right|}, \\
\mathrm{DSC}=\frac{2\left|M_{i} \cap N_{i}\right|}{\left|M_{i}\right|+\left|N_{i}\right|},
\end{gathered}
$$

where $M_{i}$ and $N_{i}$ represent the parts of category $i$ in the standard segmentation image and the algorithm segmentation image, respectively.

2.4. Statistical Analysis. SPSS20.0 statistical software was used for analysis, measurement data were expressed as mean \pm standard deviation, and $\mathrm{FA}$ and $\mathrm{MK}$ values were tested by paired $t$-test. If $P<0.05$, the difference was statistically obvious.

\section{Results}

3.1. Segmentation Results of Brain Diffusion Kurtosis ImagingBased Image Segmentation Algorithm. Image data from simulated brain database (SBD) were employed to evaluate the performance of the segmentation algorithm. The database images had proton density (PD) weighted, T1-weighted, and T2-weighted sequence patterns. In this study, the image size was $180 \times 217$, T1-weighted image was applied, slice thickness was $1 \mathrm{~mm}$, nonuniformity of intensity was $50 \%$, and noise level was 9\%. JS and DSC indexes were applied to quantitatively evaluate the performance of the image segmentation algorithm. The segmentation results of FCM, FLICM, SOM + GA, and the algorithm in this study are shown in Table 1. The JS coefficients of the algorithm proposed in this study for the segmentation of WM, GM, and CSF were $0.8408,0.7417$, and 0.6882 , respectively, and the DSC coefficients for the segmentation of WM, GM, and CSF were $0.9106,0.8507$, and 0.8025 , respectively. Therefore, the JS and DSC indexes of WM, GM, and CSF in this algorithm were all the best compared with FCM, FLICM, and $\mathrm{SOM}+\mathrm{GA}$, indicating that the segmentation accuracy of this algorithm was better than other traditional algorithms.

3.2. Comparison on FA Values of WM in the Different Parts. The FA values of patients with HIE were compared at the acute phase (2-7 days) and the chronic phase (2-3 months), and the results are shown in Figures 1-3. There were statistically great differences of FA values at the acute phase and chronic phase in the area around lesions $(0.421 \pm 0.065$ vs. $0.454 \pm 0.052$ ), posterior limb of internal capsule on the affected side $(0.498 \pm 0.027$ vs. $0.504 \pm 0.046)$, and pedunculus cerebri on the affected side $(0.558 \pm 0.038$ vs. $0.568 \pm 0.042)(P<0.05)$. However, FA values at the acute phase and chronic phase were compared in the genu of the corpus callosum $(0.482 \pm 0.054$ vs. $0.518 \pm 0.053)$, the body part of corpus callosum $(0.531 \pm 0.062$ vs. $0.539 \pm 0.042)$, the splenium of corpus callosum $(0.538 \pm 0.052$ vs. $0.528 \pm 0.038)$, and the centrum semiovale on the affected side $(0.387 \pm 0.028$ vs. $0.387 \pm 0.028)$, the corona radiata on the affected side $(0.492 \pm 0.025$ vs. $0.483 \pm 0.025)$, the centrum semiovale on the normal side $(0.395 \pm 0.033$ vs. $0.399 \pm 0.041)$, the corona radiata on the normal side $(0.508 \pm 0.036$ vs. $0.509 \pm 0.036)$, and the posterior limb of internal capsule on the normal side $(0.507 \pm 0.029$ vs. $0.501 \pm 0.034)$, and the pedunculus cerebri on the normal side $(0.564 \pm 0.044$ vs. $0.568 \pm 0.039)$, and the differences were not statistically obvious $(P>0.05)$. Except for the three parts of the splenium of corpus callosum, the corona radiata on the affected side, and the posterior limb of internal capsule on the normal side, the FA value at the chronic phase of WM in patients with HIE was higher than that at the acute phase.

3.3. Comparison on $M K$ Values of $W M$ in the Different Parts. The MK values of patients with HIE were compared between the acute stage (2-7 days) and the chronic stage (2-3 months), and Figures $4-6$ reveal the results. The MK values at the acute phase and chronic phase were compared in the area around lesions $(1.362 \pm 0.098$ vs. $1.407 \pm 0.077)$, the centrum semiovale on the affected side $(1.305 \pm 0.102$ vs. $1.343 \pm 0.076)$, the posterior limb of internal capsule on the affected side $(1.338 \pm 0.543$ vs. $1.382 \pm 0.076)$, and the pedunculus cerebri on 
TABle 1: Performance comparison of different image segmentation algorithms.

\begin{tabular}{lcccccc}
\hline & \multicolumn{3}{c}{ JS } & & DSC \\
& WM & GM & CSF & WM & GM & CSF \\
\hline FCM & 0.7567 & 0.7313 & 0.6427 & 0.8614 & 0.8389 & 0.7736 \\
FLICM & 0.8325 & 0.7402 & 0.6423 & 0.9102 & 0.8506 & 0.7910 \\
SOM + GA & 0.8373 & 0.7406 & 0.6675 & 0.9106 & 0.8507 & 0.8025 \\
$\begin{array}{l}\text { Algorithm in } \\
\text { this study }\end{array}$ & 0.8408 & 0.7417 & 0.6882 & 0.9137 & 0.8518 & 0.8156 \\
\hline
\end{tabular}

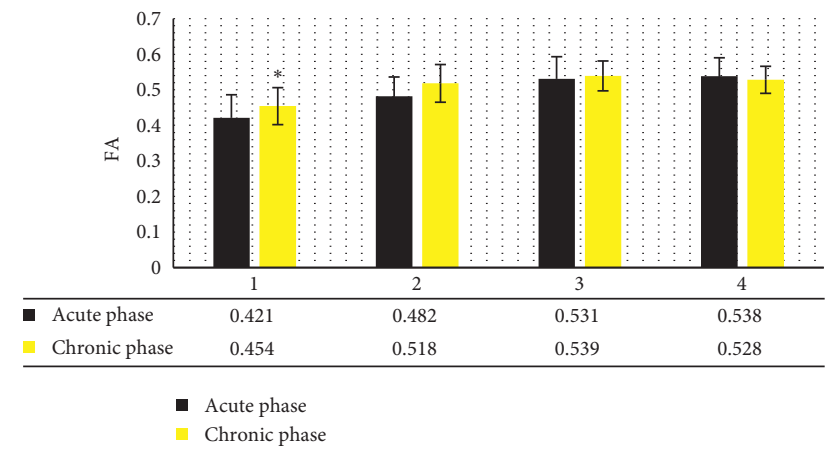

FIgUre 1: Comparison on FA values of WM in the different parts (I). Note: 1-4 stand for the area around the lesions, genu of corpus callosum, body part of corpus callosum, and splenium of corpus callosum, respectively; and * means that the difference was statistically considerable compared with the acute phase $(P<0.05)$.

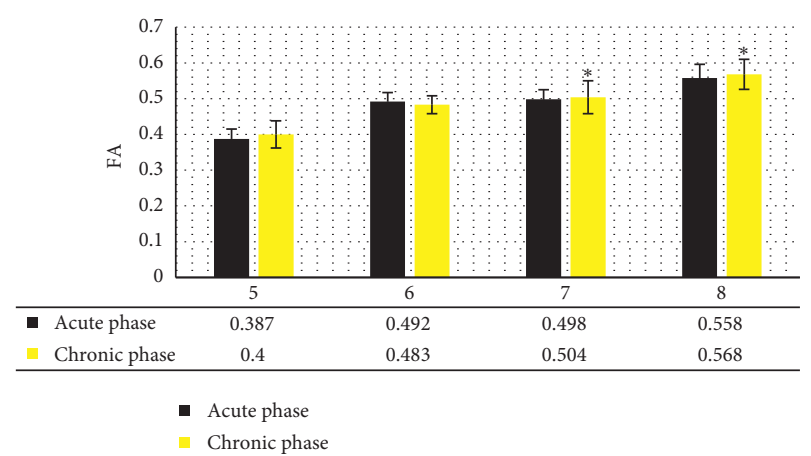

Figure 2: Comparison on FA values of WM in the different parts (II). Note: 5-8 stand for the centrum semiovale on the affected side, corona radiate on the affected side, body part of corpus callosum on the affected side, and the pedunculus cerebri on the affected side, respectively; and * expresses that there was a statistically considerable difference in contrast to the acute phase $(P<0.05)$.

the affected side $(1.329 \pm 0.089$ vs. $1.398 \pm 0.099)$, and the differences were statistically obvious $(P<0.05)$. However, there were no statistically huge differences in $\mathrm{MK}$ values at the acute phase and chronic phase in the genu of the corpus callosum (1.168 \pm 0.108 vs. $1.176 \pm 0.109)$, the body part of corpus callosum ( $1.203 \pm 0.127$ vs. $1.228 \pm 0.122)$, the splenium of corpus callosum ( $1.206 \pm 0.113$ vs. $1.231 \pm 0.089)$, the corona radiata on the affected side $(1.376 \pm 0.154$ vs. $1.452 \pm 0.078)$, and the centrum semiovale on the normal side $(1.242 \pm 0.098$ vs. $1.274 \pm 0.097)$, the corona radiata on the normal side $(1.483 \pm 0.112$ vs. $1.494 \pm 0.116)$, and the posterior limb of

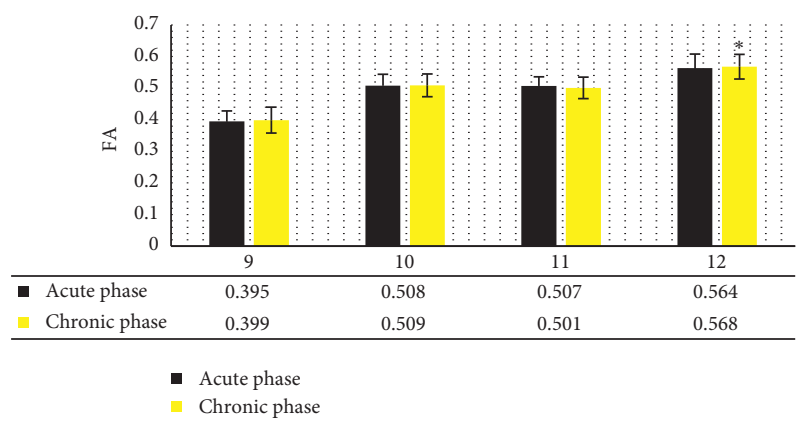

Figure 3: Comparison on FA values of WM in the different parts (III). Note: 9-12 represented the centrum semiovale on the normal side, corona radiate on the normal side, posterior limb of internal capsule on the normal side, and pedunculus cerebri on the normal side, respectively.

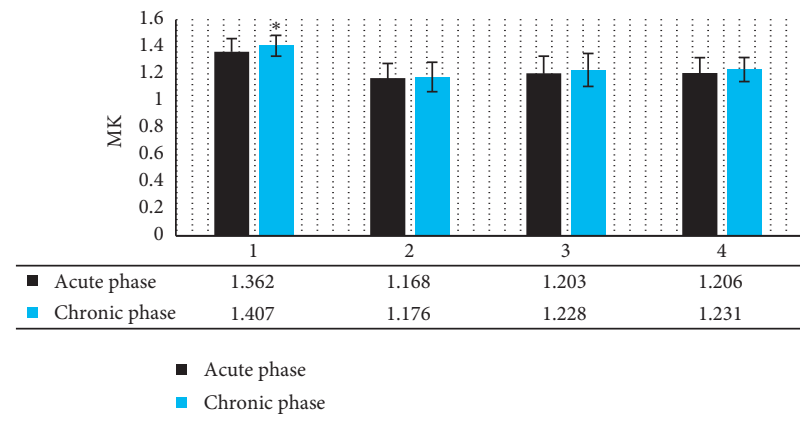

FIgUre 4: Comparison on MK values of WM in the different parts (I). Note: 1-4 stand for the area around the lesions, genu of corpus callosum, body part of corpus callosum, and splenium of corpus callosum, respectively; and ${ }^{*}$ means that the difference was statistically obvious compared to the acute phase $(P<0.05)$.

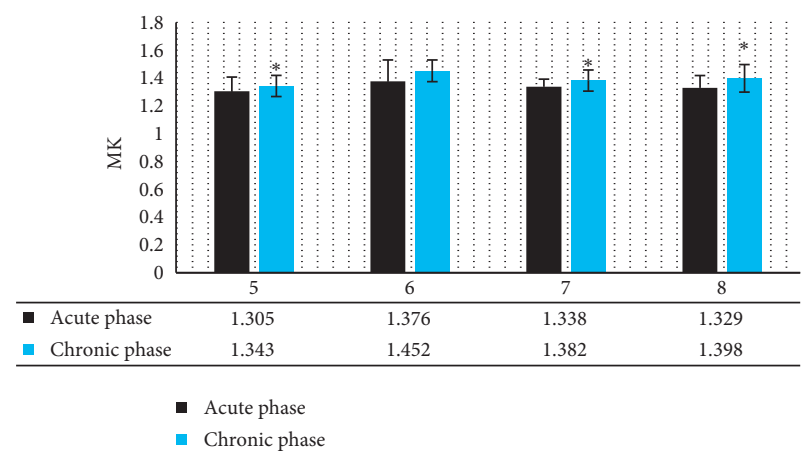

FIgURE 5: Comparison on MK values of WM in the different parts (II). Note: 5-8 stand for the centrum semiovale on the affected side, corona radiate on the affected side, body part of corpus callosum on the affected side, and the pedunculus cerebri on the affected side, respectively; and * expresses that there was a statistically marked difference in contrast to the acute phase $(P<0.05)$.

internal capsule on the normal side $(1.359 \pm 0.087$ vs. $1.390 \pm 0.096$ ), and the pedunculus cerebri on the normal side $(1.398 \pm 0.105$ vs. $1.394 \pm 0.107)(P>0.05)$. Except for the pedunculus cerebri on the normal side, the MK value at the chronic phase of WM in patients with HIE was higher than that at the acute phase. 


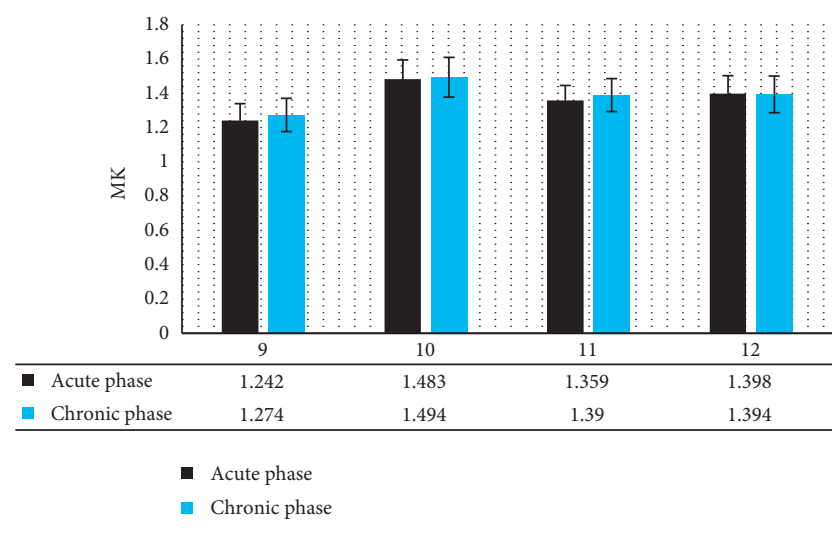

FIGURE 6: Comparison on MK values of WM in the different parts (III). Note: 9-12 expresses the centrum semiovale on the normal side, corona radiate on the normal side, posterior limb of internal capsule on the normal side, and pedunculus cerebri on the normal side, respectively.

\section{Discussion}

The brain DKI-based image segmentation algorithm was applied to the DKI image processing, and the results showed that the JS and DSC of this algorithm were the best for segmentation of brain WM, GM, and CSF. The segmentation accuracy was better than that of other algorithms. It was consistent with the research results of Chen et al. [14], which showed that the FLICM algorithm combined with random forest classifier could improve the medical image segmentation accuracy.

Hypoxic-ischemic brain damage is the main cause of death in children, with high mortality and morbidity, including cerebral palsy, epilepsy, and cognitive impairment [3]. WM lesions include the cystic and noncystic (with focal necrosis) ventricular leukomalacia and nonnecrotic diffuse WM damage. Multiple causes are associated with these damages. The anatomical and physiological characteristics of periventricular vascular structures result in WM to be liable to cerebral ischemia, to interact with infection/inflammatory factors, to activate microglia, to produce oxidative stress, proinflammatory cytokines, and glutamate toxicity, and to have energy failure and vascular integrity impairment. Hypoxicischemia may also produce selective neuronal necrosis in different brain regions [15]. Besides, DKI is further optimized on the basis of DTI. The research results of Gao et al. [16] suggested that DKI showed higher specificity for the microstructure changes of WM, which was better than DTI index, and could be applied in the diagnosis of leukoencephalopathy and HIE. In this study, DKI technology was applied to the structural remodeling of WM in patients with HIE. It was found that there were statistically marked differences in the FA and $\mathrm{MK}$ values of the area around lesions, the posterior limb of internal capsule on the affected side, and the pedunculus cerebri on the affected side, and in the MK value of the centrum semiovale on the affected side $(P<0.05)$. This was in accord with the research findings of Li et al. [17], and they employed DTI to scan 10 full-term babies without brain damage and 22 full-term babies with HIE. It was found that there were different FA, volume of voxel, and number of fiber tracts in some brain regions between the babies with brain damage and those without brain damage. The correlation between FA and neonatal behavioral neurological assessment score was most consistent with the results of the posterior limb of internal capsule. There was a reliable recombination of WM in corpus callosum in relevant experiments [18]. However, there was no statistically remarkable change in the MK and FA values of the corpus callosum $(P>0.05)$, which might be related to the course of disease and the location of lesion. In addition, the causes should be further explored and verified. MK value of the centrum semiovale on the affected side was statistically obvious $(P<0.05)$ in this study, but there was no statistically huge difference in the FA value $(P>0.05)$, indicating that DKI was more sensitive to diagnosis than DTI.

\section{Conclusion}

The brain image segmentation algorithm based on DKI was applied in the research of WM structural remodeling in patients with HIE. Comparative analysis showed that both FA value and MK value in the acute and chronic phases around the lesion, the hindlimb of the internal capsule on the affected side, and the pedunculus cerebri of the affected side were statistically different. Thus, the WM structural remodeling might exist, and the changes of FA and MK were correlated with WM structural remodeling, showing that DKI technology had great significance to the research on WM structural remodeling. However, there were still some deficiencies in this study. For example, the number of samples was limited, and the brain structure features were applied only to the $\mathrm{H}$-shaped region of CSF. In the future, the algorithm should be further optimized, and the brain structure characteristics that could be combined with the algorithm should be explored to increase the number of samples, so as to further analyze the structural remodeling of WM in patients with HIE. To sum up, the results of this study could provide reference for imaging diagnosis and clinical treatment of HIE.

\section{Data Availability}

The data used to support the findings of this study are available from the corresponding author upon request.

\section{Conflicts of Interest}

The authors declare no conflicts of interest.

\section{Acknowledgments}

This work was supported by the National Natural Science Foundation of China, no. 81771663, and Natural Science Foundation of Liaoning Province.

\section{References}

[1] Q. Wang, H. Lv, L. Lu, P. Ren, and L. Li, "Neonatal hypoxicischemic encephalopathy: emerging therapeutic strategies based on pathophysiologic phases of the injury," Journal of 
Maternal-Fetal and Neonatal Medicine, vol. 32, no. 21, pp. 3685-3692, 2019.

[2] E. P. Yıldız, B. Ekici, and B. Tatl1, "Neonatal hypoxic ischemic encephalopathy: an update on disease pathogenesis and treatment," Expert Review of Neurotherapeutics, vol. 17, no. 5, pp. 449-459, 2017.

[3] G. Wassink, J. O. Davidson, S. K. Dhillon et al., "Therapeutic hypothermia in neonatal hypoxic-ischemic encephalopathy," Current Neurology and Neuroscience Reports, vol. 19, no. 2, p. 2, 2019.

[4] C. Amarnath, T. Helen Mary, A. Periakarupan, K. Gopinathan, and J. Philson, "Neonatal parechovirus leucoencephalitis- radiological pattern mimicking hypoxic-ischemic encephalopathy," European Journal of Radiology, vol. 85, no. 2, pp. 428-434, 2016.

[5] C.-Y. Yeh, C.-M. Yeh, T.-H. Yu, K.-H. Chang, C.-C. Huang, and K.-S. Hsu, "Neonatal dexamethasone treatment exacerbates hypoxia/ischemia-induced white matter injury," Molecular Neurobiology, vol. 54, no. 9, pp. 7083-7095, 2017.

[6] D. M. Lindberg, N. V. Stence, J. A. Grubenhoff et al., "Feasibility and accuracy of fast MRI versus CT for traumatic brain injury in young children," Pediatrics, vol. 144, no. 4, Article ID e20190419, 2019.

[7] S. Currie, N. Saleem, J. A. Straiton, J. Macmullen-Price, D. J. Warren, and I. J. Craven, "Imaging assessment of traumatic brain injury," Postgraduate Medical Journal, vol. 92, no. 1083, pp. 41-50, 2016.

[8] S. Shahab, L. Stefanik, G. Foussias, M.-C. Lai, K. K. Anderson, and A. N. Voineskos, "Sex and diffusion tensor imaging of white matter in schizophrenia: a systematic review plus metaanalysis of the corpus callosum," Schizophrenia Bulletin, vol. 44, no. 1, pp. 203-221, 2018.

[9] J. H. Jensen, E. T. McKinnon, G. R. Glenn, and J. A Helpern, "Evaluating kurtosis-based diffusion MRI tissue models for white matter with fiber ball imaging," NMR In Biomedicine, vol. 30 , no. 5 , p. $10,2017$.

[10] L. Liu, M. Ma, and J. Cui, "A novel model-based on FCM-LM algorithm for prediction of protein folding rate," Journal of Bioinformatics and Computational Biology, vol. 15, no. 4, Article ID 1750012, 2017.

[11] Y. Wang, X. Liu, and L. Xiang, "GA-based membrane evolutionary algorithm for ensemble clustering," Computational Intelligence and Neuroscience, vol. 2017, Article ID 4367342, 2017.

[12] J. Cao, L. Chen, M. Wang, and Y Tian, "Implementing a parallel image edge detection algorithm based on the otsucanny operator on the hadoop platform," Computational Intelligence and Neuroscience, vol. 2018, Article ID 3598284, 12 pages, 2018.

[13] M. Lavanya and P. M. Kannan, "Lung lesion detection in CT scan images using the fuzzy local information cluster means (FLICM) automatic segmentation algorithm and back propagation network classification," Asian Pacific Journal of Cancer Prevention: Asian Pacific Journal of Cancer Prevention, vol. 18, no. 12, pp. 3395-3399, 2017.

[14] M. Chen, Q. Yan, and M. Qin, "A segmentation of brain MRI images utilizing intensity and contextual information by Markov random field," Computer assisted surgery (Abingdon, England), vol. 22, no. 1, pp. 200-211, 2017.

[15] A. Cerisola, F. Baltar, C. Ferrán, and E. Turcatti, "Mecanismos de lesión cerebral en niños prematuros [Mechanisms of brain injury of the premature baby]," Medicina, vol. 79, no. 3, pp. 10-14, 2019.
[16] J. Gao, X. Li, Y. Li et al., "Differentiating T2 hyperintensity in neonatal white matter by two-compartment model of diffusional kurtosis imaging," Scientific Reports, vol. 6, no. 1, Article ID 24473, 2016.

[17] H. X. Li, X. Feng, Q. Wang, X Dong, M Yu, and W. J Tu, "Diffusion tensor imaging assesses white matter injury in neonates with hypoxic-ischemic encephalopathy," Neural regeneration research, vol. 12, no. 4, pp. 603-609, 2017.

[18] W. Li, D. An, X. Tong et al., "Different patterns of white matter changes after successful surgery of mesial temporal lobe epilepsy," NeuroImage: Clinical, vol. 21, Article ID 101631, 2019. 